\title{
Financiamiento por Desempeño en Chile: Análisis Conceptual de un Instrumento para la Educación Superior
}

\author{
Carmen A. Araneda-Guirriman y Liliana M. Pedraja-Rejas \\ Centro de Estudios CEUTA, Universidad de Tarapacá. Casilla 7-D Arica, Chile. \\ (e-mail: caraneda@uta.cl; Ipedraja@uta.cl)
}

Recibido Sep. 25, 2015; Aceptado Nov. 23, 2015; Versión final Dic. 15, 2015, Publicado Jun. 2016

\begin{abstract}
Resumen
El presente trabajo muestra un análisis conceptual respecto a los cambios que han ido experimentando las instituciones de educación terciaria en términos de su financiamiento, tanto a nivel mundial como a nivel nacional. Se visualiza que estos cambios se encuentran fundamentados en la masificación, privatización e internacionalización de la educación superior, requiriéndose que éstas sean cada vez eficientes y eficaces en la utilización de sus recursos. Las nuevas estrategias establecidas para el otorgamiento de recursos a las instituciones de educación superior, están basadas en la competitividad y la evaluación, incorporando elementos tales como la rendición de cuentas públicas y la evaluación del desempeño. Se concluye que es necesario revisar el instrumento para el otorgamiento de recursos a las universidades chilenas denominado convenio de desempeño y que estos cambios requieren de un nuevo tipo de relación entre las instituciones de educación superior y el Estado.
\end{abstract}

Palabras clave: financiamiento; educación superior; desempeño académico; rendición de cuentas; competitividad

\section{Performance-based Funding in Chile: Theoretical Framework Analysis for Financial Instrument in Higher Education Institutions}

\begin{abstract}
This paper presents a theoretical framework about the changes that higher education institutions have been experiencing in terms of its financing, both worldwide and locally in Chile. It is shown that these changes are based in the massification, privatization and internationalization of higher education, requiring that they must be increasingly efficient and effective in the use of their resources. The new strategies established for the granting of resources to higher education institutions, are related to competitiveness and evaluation, including elements such as public accountability and performance evaluation. It is concluded that it is of paramount importance to review the instrument for granting resources to Chilean universities, named performance agreement, and these changes require a new kind of relationship between higher education institutions and the State.
\end{abstract}

Keywords: funding; higher education; academic performance; accountability; competitiveness 


\section{INTRODUCCIÓN}

Las consecuencias de la crisis financiera internacional del 2008/09 y la actual crisis económica a nivel global, han puesto bajo una enorme presión, como era de esperar, a los fondos públicos destinados a la educación superior. Junto con ello, durante los últimos 20 años, se han venido introduciendo una serie de métodos dirigidos a la gestión eficaz de los costos, los cuales han sido importados desde el sector de los negocios, situación que ha empujado a las universidades a buscar nuevas fuentes de ingresos, ya que la magnitud de las reducciones recientes en países como Reino Unido, Estados Unidos o Chile tienen el potencial de transformar la esencia misma de la educación superior pública (Lebeau, 2012).

En efecto, durante los últimos años, los regímenes de responsabilidad basados en el rendimiento se han vuelto cada vez más frecuentes en los gobiernos, lo cual ha traído consigo que Estados, como Finlandia y Estados Unidos hayan adoptado políticas de financiamiento que buscan vincular la entrega de recursos financieros institucionales con medidas objetivas de desempeño, como lo es el caso de las universidades americanas que han luchado por satisfacer la demanda de mejorar su desempeño (Rabovsky, 2012). Asimismo, uno de los cambios más profundos en la educación superior durante las últimas décadas es el aumento del interés por la rendición de cuentas (Accountability). A este respecto se debe sumar el hecho de que dada la escases de los recursos obliga a diversos sectores de la sociedad, no solo del ámbito educativo, a competir por estos, lo que conlleva necesariamente a rendir los recursos recibidos (PedrajaRejas y Rodríguez-Ponce, 2015).

Este interés se debe a un número de diversos factores, entre los cuales se destaca la masificación de la educación superior, situación que ha incrementado el gasto y el interés en el sector, orientando las preocupaciones hacia la forma en que los recursos son usados y el valor del dinero invertido. Por tanto, una política fuerte junto con las creencias ideológicas relacionadas al surgimiento de la sociedad del conocimiento, han contribuido a definir la atención sobre la educación superior como un elemento clave que va soportando estas transformaciones (Stensaker y Harvey, 2011). En este contexto, se puede destacar que en Estados Unidos, muchos estados han comenzado a adoptar la estrategia de financiamiento por desempeño durante los años 90, a causa del movimiento de rendición de cuentas (Dougherty y Reddy, 2013).

Por consiguiente, se puede establecer que los mensajes de la política y de las políticas públicas identificados para el periodo de 1987 a 2003 en el caso de Reino Unido, junto con valorar las contribuciones hechas por la educación superior al logro de objetivos económicos y sociales, están orientados al incremento de la eficiencia en las universidades, junto con la necesidad de encontrar nuevos recursos de ingreso y mejorar su desempeño en toda la gama de sus actividades y servicios. Más aún, en los últimos 10 años ha sido necesario mirar los cambios en los patrones de gobernanza al interior de las universidades (Middlehurst, 2013).

Junto con lo anterior se deben considerar las transformaciones que ha experimentado la educación superior chilena durante el último siglo, las cuales se configuran como un complejo entramado de variaciones que son continuas y permanentes en el tiempo y que se encuentran determinadas exógenamente, por cambios en las esferas de la educación (Brunner, 2015). Siendo precisamente una de éstos, el sistema de financiamiento de la educación superior, ya que hoy en día prima un régimen de financiación que incentiva la generación de ingresos por parte de las propias instituciones de educación superior basado en el cobro de aranceles y aportes selectivos del Estado, entre otros (Brunner, 2004). Por lo tanto, resulta vital con el fin de contribuir al acervo de conocimientos respecto al financiamiento de la educación superior, partir con una reflexión y análisis de la bibliografía existente respecto al estado del arte actual en lo que a financiamiento y educación superior se requiere, haciendo énfasis en los desafíos que contempla la rendición de cuentas públicas y la evaluación por desempeño al cual deben ser sometidas las instituciones de educación terciaria.

En este sentido, vale precisar, que en la actualidad las políticas de financiamiento de educación superior en Chile están en discusión y definición, por tanto, es un tema en desarrollo, lo cual evidencia el carácter evolutivo y de cambio del sistema en general.

\section{FINANCIAMIENTO EN LA EDUCACIÓN SUPERIOR}

Durante los últimos años, los gobiernos han focalizado la definición de sus políticas en el desempeño con el fin de influir en el comportamiento las instituciones de Educación Superior. No obstante, la mayoría de los estudios de impacto indican que estas políticas han tenido un efecto limitado en el rendimiento de estas instituciones, donde gran parte de este fracaso se explica sobre la base de la teoría del agente principal, teoría de la dependencia y el neoinstitucionalismo, por tanto, estos análisis tienden a ver a las universidades de manera aislada de su ecología mundial real (Nisar, 2015). 
Por tanto, abordar el tema del financiamiento en las universidades resulta trascendental, debido a que se configura como un elemento catalizador del funcionamiento de una institución, siendo fundamental para la supervivencia de éstas, vinculándose además fuertemente a la calidad del cuerpo docente que la conforma (Araneda-Guirriman et al., 2013). Asimismo, al existir más universidades, más estudiantes y mayor diversidad de temas, se tiene como resultado que las características y los costos de los diferentes grados académicos en distintas instituciones varíe ampliamente, y que por tanto, las universidades necesiten tener un financiamiento diferenciado, lo cual en la práctica es muy difícil, en consecuencia, un sistema de masas en un mundo complejo requiere de mecanismos de financiamientos que permitan a instituciones cobren variados precios a diferentes costos y misiones (Barr, 2004).

Las modalidades de financiamiento son variadas, aunque para la mayoría de los países, el Estado ha sido tradicionalmente el principal financiador de la educación superior En este sentido, la masificación ha depositado grandes tensiones en el financiamiento estatal, y en todos los casos, los gobiernos ya no creen ser capaces de poder financiar adecuadamente la educación superior de masas (Altbach, 2013). Más atención aún se debe poner en el financiamiento de la educación superior pública, el cual ha sido drásticamente disminuido, las tasas se han incrementado, junto con la fuerza política que fuerza cada vez más estrictos presupuestos de austeridad que amenazan con ser cada vez aún más reducidos (Reiff, 2014).

Por otra parte, en Europa gran parte de las universidades públicas son financiadas principalmente por el dinero de los contribuyentes y regulado por el Estado a través de las leyes de educación superior. A partir de estas características organizativas se pueden deducir los siguientes factores y condiciones de contexto con un alto grado de relevancia para todos los actores involucrados en la gobernanza de la universidad, no solo para los miembros del consejo, particularmente en la dependencia financiera de las universidades públicas en Europa, las cuales son corporaciones públicas, es decir, ellas son controladas por el Estado. Contrario a los cuerpos corporativos de administración de los directores, los consejos universitarios no tendrán el mismo espacio para maniobrar debido a la dependencia financiera con el Estado, que implica mecanismos o fórmulas de financiación predefinidos (Kretek et al., 2013), como sucede con los convenios de desempeño en el año 2015. A este respecto, se puede hablar de un cambio de política, donde la autonomía es redefinida como una nueva independencia organizacional de las universidades, tanto como actor estratégico como destinatario del control gubernamental (Enders et al., 2013).

En lo que respecta a Chile, el gasto público para las instituciones de educación terciaria ha obtenido principalmente por tres vías: el Aporte Fiscal Directo (AFD), el cual es exclusivo para las universidades pertenecientes al Consejo de Rectores de Universidades Chilenas ( $\mathrm{CRUCH})$ el Aporte Fiscal Indirecto y los fondos asociados a desempeño (Paredes, 2015).

De esta forma, el financiamiento de las instituciones universitarias se sustenta principalmente por el Aporte Fiscal Directo (AFD), el cual consiste en un subsidio de libre disponibilidad asignado en un $95 \%$ basado en criterios históricos, y el $5 \%$ restante de acuerdo con indicadores de desempeño anuales vinculados a la matrícula estudiantil, el número de académicos con postgrado y el número de proyectos y publicaciones de investigación de excelencia, que se encuentra regulado mediante el DFL $N^{\circ} 4$ de 1981, junto con el Decreto $\mathrm{N}^{\circ} 128$ y sus modificaciones (MINEDUC, 2015a). A pesar de que según la tabla 1 se evidencia un leve aumento en los recursos anuales asignados, sin embargo, es posible observar que la contribución del Estado al presupuesto basal de las instituciones universitarias que forman parte del $\mathrm{CRUCH}$ ha ido disminuyendo en el tiempo (Wörner, 2014).

De igual modo, se debe consignar que existe en este instrumento AFD una desigualdad de origen desventajosa, particularmente para las universidades regionales, con lo que se establece el comienzo de una clasificación y segmentación del sistema que se mantiene hasta el día de hoy (Améstica et al., 2014), lo cual se puede evidenciar en la siguiente tabla:

Tabla 1. Aporte Fiscal Directo Universidades del CRUCH 2010-2015.

\begin{tabular}{|l|c|c|c|c|c|c|}
\hline & $\begin{array}{c}2010 \\
(95 \%)\end{array}$ & $\begin{array}{c}2011 \\
(95 \%+5 \%)\end{array}$ & $\begin{array}{c}2012 \\
(95 \%+5 \%)\end{array}$ & $\begin{array}{c}2013 \\
(95 \%+5 \%)\end{array}$ & $\begin{array}{c}2014 \\
(95 \%+5 \%)\end{array}$ & $\begin{array}{c}2015 \\
(95 \%+5 \%)\end{array}$ \\
\hline Total AFD UCH y PUC & 47.622 .852 & 50.619 .183 & 53.966 .325 & 57.483 .742 & 61.086 .264 & 63.905 .998 \\
\hline UTA & 5.267 .724 & 6.173 .739 & 6.802 .306 & 7.409 .431 & 8.202 .951 & 8.989 .979 \\
\hline UBB & 3.009 .642 & 3.482 .769 & 3.872 .269 & 4.352 .976 & 4.940 .071 & 5.484 .081 \\
\hline Total AFD & 143.253 .508 & 155.769 .342 & 168.153 .005 & 181.681 .627 & 196.488 .679 & 208.277 .999 \\
\hline
\end{tabular}


En efecto, de acuerdo a la tabla 1 se puede observar que durante los últimos 5 años sólo 2 instituciones universitarias concentran alrededor del $30 \%$ del aporte fiscal directo, lo cual es muy superior a lo que reciben las universidades regionales, como por ejemplo la Universidad de Tarapacá y Universidad del BíoBío, debido a que no logran superar el $5 \%$ de los aportes recibidos.

Por otra parte, se encuentra el Aporte Fiscal Indirecto (AFI), otorgado a todas las instituciones de educación superior en función del puntaje (PSU) logrado por los estudiantes que ingresan a ellas y es además de libre disposición, por lo tanto, se encuentra en la base de funcionamiento de las universidades, porque se considera un ingreso anual estable que no tiene gran variabilidad año a año (Williamson y Sánchez, 2009).

No obstante, la estabilidad y aumento gradual cercano al $1 \%$ que ha tenido anualmente el AFI y que se puede observar en la tabla 2, resulta útil indicar que este instrumento es bastante bajo para las universidades en regiones, donde puede llegar al $0,3 \%$ del monto anual distribuido, como sucede para la Universidad de Tarapacá, y donde dos universidades de la Región Metropolitana acaparan el $50 \%$ de los recursos, evidenciándose de esta forma falencias e insuficiencia de recursos para las instituciones de regiones, lo cual ha generado una segregación económica como resultado (Améstica et al., 2014).

Tabla 2. Aporte Fiscal Indirecto 2011-2015 Universidades del CRUCH

\begin{tabular}{|l|l|l|l|l|l|}
\hline & 2011 & 2012 & 2013 & 2014 & 2015 \\
\hline UCH y PUC & 8.505 .292 & 8.642 .901 & 9.304 .484 & 9.341 .099 & 9.716 .470 \\
\hline UBB & 126.070 & 132.225 & 124.413 & 156.943 & 148.792 \\
\hline UTA & 45.216 & 45.649 & 31.070 & 36.903 & 36.410 \\
\hline TOTAL AFI UES CRUCH & 16.910 .319 & 17.138 .754 & 17.231 .145 & 17.941 .211 & 18.219 .959 \\
\hline
\end{tabular}

A juicio de Flores et al. (2011) este instrumento presenta deficiencias asociadas a la equidad del sistema de educación terciaria, ya que plantea un sesgo contra los estudiantes de menor nivel socioeconómico, al sustentar su distribución en base a los puntajes obtenidos por los estudiantes hereda el sesgo en contra de los estudiantes de nivel socioeconómico menor, llevando a que la gran mayoría de los causantes sean los alumnos de los mayores quintiles de ingreso.

A pesar de lo indicado anteriormente, es necesario señalar que el gasto público en educación superior en Chile evidenció un incremento para el periodo 2009-2013, pasando desde un $14,6 \%$ a un $34,6 \%$, no obstante aquello, Chile se encontró entre los tres países con el valor más bajo, posicionándose lejos del $70 \%$ de gasto público que evidenció el promedio de la OCDE (MINEDUC, 2015a). En efecto, abordar el tema del financiamiento es complejo de abordar dado los diferentes niveles de análisis que se pueden establecer, como sucede con los datos generales analizados previamente.

\section{FINANCIAMIENTO POR DESEMPEÑO}

En este contexto de financiamiento de la educación terciaria, una de las más populares tendencias de políticas que ha surgido en la educación superior, es el financiamiento por desempeño (Hillman et al., 2015). En efecto, el financiamiento por desempeño es una respuesta al incremento del número de Estados que están usando la comunicación y la potencial influencia de los resultados de educación superior que van para el aprendizaje y el logro de tasas de graduación (Dougherty y Reddy, 2013). Es así como en distintos países, tales como Suecia y Noruega, el financiamiento fiscal de las instituciones de educación superior se ha convertido en parte dependiente del desempeño en la docencia y la investigación (Pettersen, 2015).

El financiamiento por desempeño es un método de financiación de las instituciones de educación superior pública, basado en resultados tales como la retención, la finalización del grado e inserción laboral y no en los inputs como la matrícula (Dougherty et al., 2013). Como lo plantea Rabovsky (2012), la lógica causal que se encuentra detrás de los mecanismos de rendición de cuentas por desempeño implica que los incentivos se reestructurarán de una forma que dé lugar a cambios en la gestión que están orientados hacia la mejora del desempeño con respecto a los resultados del cliente. No obstante lo anterior, gran parte de la investigación que analiza el impacto de estas políticas, particularmente en el área de la educación superior, se salta partes de la cadena causal, centrándose de manera exclusiva en analizar el impacto que han tenido la adopción de las políticas de desempeño en los resultados de éxito académico de los estudiantes. Sumado a lo anterior, se debe tener en consideración que en los primeros intentos para medir la eficiencia y el desempeño institucional en general se han encontrado con la resistencia pasiva o negligencia benigna en los círculos académicos (Alexander 2000). Asimismo, se debe señalar que las demandas externas por la rendición de cuentas se configuran como un gran incentivo por el fortalecimiento de la capacidad de gestión (Arata y Rodríguez, 2009). 
Por tanto, el financiamiento basado en desempeño es una estrategia de distribución de los recursos utilizados en educación superior para recompensar a los proveedores de esos servicios por los resultados que ellos han logrado. Las instituciones universitarias que producen resultados superiores a la media ganan financiamiento adicional, mientras que las que no alcanzan ese valor pueden enfrentar reducciones de fondos y pueden ser objeto de asistencia técnica para abordar sus deficiencias de desempeño. De esta forma, los esfuerzos para introducir el financiamiento basado en el desempeño en la educación a menudo se encuentran con la resistencia de los administradores del programa y su personal, que, entre otras objeciones, manifiesten el miedo a la aplicación del financiamiento competitivo puede penalizar injustamente a aquellos estudiantes de bajo rendimiento académico (Klein, 2015). Complementando lo anterior, se puede establecer además que los sistemas de educación superior poseen variados costos asociados como resultado de la masificación de ingreso que ha obligado incrementar recursos dentro de las cuales se encuentra la infraestructura física, cuerpo docente y a presión por mejorar la calidad y la pertinencia de las actividades académicas y de generación de conocimiento (Brunner et al., 2005).

Los convenios de desempeño se utilizan, por ende, siempre junto con otros instrumentos de la política, es decir podrían estar relacionados con modelos de financiamiento basado en rendimiento. En algunos países existe una relación directa entre los objetivos fijados en los convenios de desempeño y el financiamiento principal asignado a las instituciones, como Finlandia y Austria. En otros países el financiamiento base y el por desempeño se abordan por separado como Luisiana (de Boer et al., 2015).

En Finlandia, a partir de la década de los noventa, se comienza a diseñar el modelo de financiamiento de una universidad estaba caracterizado por el principio de la gestión de resultados, para lo cual, el Ministerio de Educación, elabora un convenio de desempeño (contrato) con cada institución sobre la base de una propuesta inicial que es efectuada con cada universidad, donde se acuerdan resultados para un periodo de tres años. En este sentido, el nivel total de financiamiento para cada universidad es incluido en este contrato (Hölttä y Malkki, 2000). De igual modo otros países de Europa, tales como Austria, República Checa, Dinamarca, Alemania e Irlanda entre otros poseen convenios de desempeños, los cuales juegan un rol crucial en la gobernanza y la gestión estratégica de los sistemas de educación superior (Strehl et al., 2007). En el caso de las universidades danesas aproximadamente el $90 \%$ del financiamiento proviene desde el Estado, del cual un $60 \%$ es basado en desempeño. Más específicamente, las universidades que son financiadas sólo para la educación, están totalmente financiadas en base a desempeño, mientras que aquellas que se orientan en la investigación básica, se sustentan en un 30\% en base a desempeño y por medio de la investigación competitiva (de Boer et al., 2015).

Por otra parte, en Estados Unidos, entre los años 1979 y 2007, 26 estados experimentaron con medidas que intentaron incorporar el desempeño institucional como un determinante para el financiamiento de la educación superior. Durante dicho periodo, 14 estados que promulgaron programas de financiamiento basado en desempeño, los descontinuaron, aunque dos de ellos han reestablecido nuevos programas. La insatisfacción de los estados se derivó del hecho de que este modelo de financiación poseía una serie de defectos de diseño, puesto que en algunos casos los programas eran inflexibles a las diferencias institucionales, teniendo como consecuencia requisitos rígidos y arbitrarios, fijándose en los resultados más que el proceso intermedio, así como también, en algunos casos no pudieron asignar suficiente financiación para crear incentivos reales para que las instituciones pudieran mejorar. Luego de este modelo inicial, y considerando los errores del pasado, el modelo de financiamiento por desempeño fue adquiriendo popularidad, siendo una tendencia que tiende a enfatizar la tendencia de recompensar los progresos y no solo en la finalización, reconocer las diferencias entre las instituciones y compartimentar los mayores porcentajes que son base para el financiamiento con el fin de incentivar el cambio transformador (Miao, 2012). Sin duda alguna, esta experiencia y evaluación puede servir para evaluar el caso chileno, sobre todo en lo que respecta rescatar el proceso intermedio de la ejecución de los convenios de desempeño chilenos.

De esta forma, para comprender por qué estas políticas funcionan o no, se debe comenzar por analizar si tiene éxito el cambio de las estructuras de incentivo que los gestores públicos deben enfrentar, puesto que si no tienen éxito al hacerlo, se rompe la lógica causal de la gestión del desempeño y los impactos deseados son poco probables que se lleven a cabo (Rabovsky 2012). Asimismo, los estándares de los procedimientos de operación en educación han sido considerablemente cuestionados, cada vez más por quienes definen las políticas, los líderes de opinión, y también por los ciudadanos comunes y corrientes, quienes están viendo la educación superior como ineficiente, caracterizada por gastos sin control, con poco trabajo por parte de los académicos y alto número de administrativos, donde a su vez se aplican débiles estándares de admisión académica, favoreciendo la investigación por sobre la docencia (Alexander, 2000). En este contexto, el financiamiento por desempeño se ha vuelto posible porque los Estados tienen disponible mejores y mayor cantidad de datos sobre el desempeño de la institución y también capacidad para vincularlo al desempeño (Alexander, 2000). 
Por otra parte, el liderazgo monetario y la capacidad del equipo de la universidad de asignar libremente es reducida, lo cual tiene un impacto en la motivación del miembro del equipo directivo a participar en los procesos de dirección quedará restringida (Kretek et al., 2013). En este sentido, se puede establecer que en la actualidad existe una presión permanente sobre dos indicadores de la gobernanza, por una parte, sobre el autogobierno gerencial, donde las instituciones públicas se rigen bajo los criterios de la Nueva Gestión Pública y por otra parte, el uso de modalidades de cuasi mercado para la asignación del financiamiento por parte del Estado (Brunner, 2015). En efecto, esta nueva gestión pretende transformar las organizaciones de gran escala en unidades menores con un control centralizado de la gerencia y la descentralización de las responsabilidades, dándose importancia en este modelo a los inputs que tenga relación con los resultados y que son medidos en relación a indicadores de control de eficacia, lo cual al ser aplicado al contexto de la esfera pública y de la educación incorpora la inclusión de valores y prácticas de mercado en la regulación y la organización de áreas que tradicionalmente ha funcionado de una manera distinta (Ganga y Valdivieso, 2013).

\section{FINANCIAMIENTO POR DESEMPEÑO EN CHILE}

Una vez analizado el financiamiento por desempeño en otros contextos cobra relevancia discutir el incipiente caso chileno desde una perspectiva conceptual. Es así como en primera instancia se puede establecer la importancia que tiene para el sistema de educación superior chileno el financiamiento por desempeño, puesto que incentivará los fondos competitivos como un enfoque coherente de financiamiento adicional a las instituciones, sobre la base de objetivos definidos vinculados con la eficacia institucional y gestionado por resultados (Middaugh et al., 2008).

En el sistema de educación superior chileno, el financiamiento público de la educación terciaria es orientado a las universidades, que son la oferta, o a los estudiantes, quienes conforman la demanda. Asimismo, la tendencia nacional en materia de criterios de asignación de recursos fiscales está, desde fines de la década de los noventa, dirigida a priorizar los fondos concursables y el financiamiento por resultados, lo cual se vincula a las tendencias internacionales de desempeño y accountability (Améstica et al., 2014). Es así como en lo referente a la experiencia que existe en Chile, respecto a la implementación de financiamiento competitivo se encuentra el instrumento del Ministerio de Educación de los convenios de desempeño se puede establecer que estos han sido gestionados y negociados con los equipos de rectoría lo que se puede interpretar como proyecto institucionales, ya que la estructura de la toma de decisiones en la organización y las prioridades de la facultad pueden configurarse como obstáculos para la sinergia y articulación que se requiere con en la ejecución de estos instrumentos de financiamiento por desempeño que requiere de la definición de estrategias que sean consonantes con los objetivos del convenio (Reich et al., 2011).

En este contexto tal como lo plantea Fernández (2015) resulta fundamental investigar la naturaleza y los impactos que tienen hoy en día los instrumentos de financiación, pues se están configurando como el elemento central de la política de financiamiento, llevando consigo a su vez un componente de negociación como sucede con estos convenios de desempeño. Ahora bien, en consonancia a lo planteado por Reich et al (2011) no perder el objetivo principal de este instrumento por desempeño es incrementar la efectividad del gasto público, alinear los objetivos institucionales con los objetivos que son relevantes en el contexto nacional, fomentar la rendición de cuentas públicas, y también vincular el mejoramiento del desempeño institucional (Reich et al., 2011).

Cabe mencionar, que, en materia de instrumentos de financiación, previa a la existencia de los convenios de desempeño se puede encontrar al Programa MECESUP (Mejoramiento de la Equidad y Calidad de la Educción Superior) el cual estaba apoyado por el Banco Mundial y comenzó a fines de la década de los 90 cuando Chile aún no tenía regulado el tema de la calidad. Este programa tenía por finalidad principal recuperar la infraestructura académica y el aseguramiento de la calidad (Reich, 2012). En el año 1999 se efectuó el primer concurso competitivo, pasando esta vez desde un sistema de asignación de recursos basados en criterios históricos, sin rendición de cuentas públicas a un nuevo escenario de asignación por resultados. Dado los buenos desempeños de esta iniciativa, durante el año 2005 se decidió continuar con los esfuerzos, estableciéndose otro convenio de préstamo con el Banco Mundial, dando de esta forma inicio al MECESUP2, donde se sentaron las bases para un sistema de financiamiento incremental basado en convenios de desempeño, reforzándose la lógica de la rendición de cuentas por el desempeño. Más aún, es importante destacar, que estas modificaciones devienen a nivel global, ya que en las últimas décadas se han llevado a cabo cambios dramáticos en la forma en que interactúan los gobiernos con las instituciones de educación superior. En efecto, una nueva motivación económica está conduciendo a los Estados a redefinir la relación, presionando a las instituciones a la rendición de cuentas, a ser más eficaces y productivos en la utilización de los recursos públicamente generados, de manera más eficiente (Alexander, 2000). 
Teniendo en consideración lo señalado anteriormente, cabe precisar, que en el caso de Chile existe muy poca evidencia sobre el financiamiento por desempeño en educación superior, al respecto se puede hacer mención al trabajo de Reich et al. (2011) en donde se realiza un análisis del instrumento Convenio de Desempeño que opera con esta lógica de rendición de cuentas y desempeño, donde se genera un contrato entre la universidad y el Estado, por medio del cual se establece un compromiso de los desempeños notables que conlleven a un mejoramiento significativo de la institución que justifique el traspaso de recursos y financiamiento, siendo un instrumento competitivo de adjudicación de recursos asociados a un alto impacto estratégico que entrega fondos en base a resultados y permite a la institución generar acciones de transformación. Cabe precisar que por gestión, tanto académica como administrativa financiera, se hace referencia a aspectos funcionales, cuya implementación contribuiría al éxito en la medida que responda a los requerimientos de la dirección estratégica, es decir, la gestión hace referencia a los pasos que se dan para completar el trayecto definido por los objetivos estratégicos de la institución (Pedraja y Rodríguez, 2014).

En relación al instrumento convenio de desempeño, se puede establecer que posee diferentes ámbitos para la asignación de los recursos, encontrándose: Humanidades, artes y ciencias sociales; formación inicial de profesores; armonización curricular; internacionalización de programas de doctorado; intercambio y movilidad académica internacional; formación técnico-profesional y apoyo a la innovación (Fernández, 2015). Asimismo, los convenios de desempeño se llevan a cabo por medio de varias etapas que son genéricas a las diferentes convocatorias según ámbito, las que pueden presentar algunas particularidades expresadas en las bases de cada concurso. Las etapas que contemplan los convenios de desempeño son las bases, propuestas, preselección, selección, negociación, tramitación, implementación y seguimiento (MINEDUC, 2015a).

En efecto, relacionado con lo señalado anteriormente está la importancia que tiene para las universidades tener una gestión institucional que resguarde la eficiencia y eficacia en su funcionamiento global, debido al impacto que esto tiene sobre la calidad de los resultados que logre la institución. Es así como la eficiencia económica de las universidades es un indicador de la gestión al interior de las universidades, que repercute en la calidad de su quehacer institucional (Rodríguez Ponce et al., 2011). Los concursos para los tres últimos ámbitos son anuales y en cada uno de ellos varía en número de instituciones beneficiadas. Por ejemplo, para el concurso 2013 en el ámbito de Educación Superior Regional, el monto total a distribución entre 6 instituciones fue de $M \$ 6.985 .888$, recibiendo cada una de ellas un monto promedio aproximado de $\mathrm{M} \$ 1.164 .314$. Cabe mencionar que el monto que recibe cada institución es para una ejecución de tres años con el fin de acelerar procesos que sin esa inyección de recursos tardarían en realizarse. Por ende, dichos recursos se alinean a los objetivos institucionales, y en consecuencia deben apalancar los recursos que tiene la institución.

Por consiguiente, y de acuerdo a los datos de la tabla 3, se puede establecer que para el año 2013 el Ministerio de Educación distribuyó, como resultados de sus concursos, un total de $M \$ 41.774 .339$, los cuales fueron destinados a universidades del $\mathrm{CRUCH}$, y universidades privadas, así como también Centros de Formación Técnica, lo cual corresponde al $24 \%$ de los fondos basales recibidos por las universidades del CRUCH, a través del AFD 2013.

Tabla 3. Recursos asociados y ámbitos de convenio de desempeño 2013.

\begin{tabular}{|l|c|l|l|}
\hline Ámbitos adjudicados & $\begin{array}{l}\text { Instituciones } \\
\text { beneficiadas }\end{array}$ & $\begin{array}{l}\text { Monto total para } \\
\text { distribución }\end{array}$ & $\begin{array}{l}\text { Monto promedio aproximado } \\
\text { recibido por las instituciones }\end{array}$ \\
\hline Educación Superior Regional & 6 & $\mathrm{M} \$ 6.985 .888$ & $\mathrm{M} \$ 1.164 .314,7$ \\
\hline Formación Inicial de profesores & 5 & $\mathrm{M} \$ 5.197 .442$ & $\mathrm{M} \$ 1.299 .360,5$ \\
\hline Innovación en la Educación Superior & 4 & $\mathrm{M} \$ 9.998 .599$ & $\mathrm{M} \$ 2.499 .649,8$ \\
\hline Innovación Académica & 5 & $\mathrm{M} \$ 8.104 .458$ & $\mathrm{M} \$ 1.620 .891,6$ \\
\hline Fortalecimiento Técnico Profesional & 5 & $\mathrm{M} \$ 3.498 .866$ & $\mathrm{M} \$ 699.773,2$ \\
\hline Planes de Mejoramiento de Programa & 47 & $\mathrm{M} \$ 7.989 .086$ & $\mathrm{M} \$ 169.980,6$ \\
\hline Total recursos por Convenio de Desempeño 2013 & & $\mathrm{M} \$ 41.774 .339$ & \\
\hline
\end{tabular}

Adicionalmente, la cantidad de recursos invertidos en la iniciativa piloto de convenios de desempeño firmados en 2007 entre el Ministerio de Educación y las Universidades de Tarapacá, de la Frontera, de Chile y del Bío-Bío muestra el impacto que tuvo dicho instrumento en las universidades que participaron de la primera experiencia, al considerar una inversión cercana a 10 billones de pesos chilenos, lo cual significó para el caso de la Universidad del Bío-Bío, por ejemplo, que estos recursos fueran casi un $30,1 \%$ del total que recibió la institución por Aporte Fiscal Directo, siendo por tanto una contribución bastante significativa para su gestión (López et al., 2011). 
Este instrumento de Convenio de Desempeño, por tanto, se puede definir como un contrato que determina el objetivo del convenio estableciendo además como compromiso: asociación entre las partes para alcanzar los resultados definidos; articulación alrededor del mejoramiento académico y de las capacidades de gestión; alineamiento explícito entre las prioridades de la institución y las ministeriales; mejoramiento notable de la gestión académica; entrega oportuna de información académica y financiera; apoyarse en una unidad de análisis institucional; disposición para la revisión y monitoreo y sistemático desde el ministerio, generar anuario con indicadores. De igual modo, el convenio obliga a la institución, a través de este contrato, al cumplimiento de las finalidades, hitos e indicadores definidos (Reich et al., 2011). En efecto, las instituciones de educación superior, por medio del convenio de desempeño, se comprometen a logros notables que signifiquen un mejoramiento en la institución, que por sí sola no habrían logrado (MINEDUC, 2015a).

De esta forma los recursos que son entregados a las instituciones mediante convenio de desempeño representan solo una parte de los recursos para financiar los objetivos (gastos elegibles), otros gastos que son discrecionales, que no son elegibles, podrán ser financiados con ingresos propios de las instituciones de educación terciaria (Reich et al., 2011).

Complementando lo anteriormente señalado, se encuentran los planteamientos de Laband y Lentz (2003) quienes analizaron la relación existente entre costos y resultados en la educación superior, en este sentido, indican que hay diferencias significativas en el costo de funcionamiento entre públicas y privadas. Asimismo, en teoría, los costos de las instituciones de educación superior sin fines de lucro se caracterizan por el aumento de los desembolsos de producción por unidad de coste (por ejemplo, por estudiante), por otra parte, las instituciones de educación superior con fines de lucro, deben maximizar los beneficios, reduciendo los costos por unidad de resultado (Laband y Lentz, 2004). En efecto, se puede establecer la coexistencia de instituciones que tienen comportamiento distinto, debido a que poseen diferentes objetivos, costos y calidad, generando una competencia multidimensional (Améstica et al., 2014). Es así como los fondos públicos son relevantes para financiar la educación superior, situación que depende de elementos la economía, tales como los costos y la productividad de los sistemas de educación terciaria, y otros aspectos que a su vez describen el proceso de formación (Viaene y Zilcha, 2011).

Respecto a esto, las decisiones que se tomen en torno a las medidas sobre el desempeño, tendrán, bajo estas circunstancias, un efecto económico directo, y la presión por financiamiento será una consecuencia del desempeño de la gestión en este sector (Pettersen, 2015). En este contexto, la idea de supervisión del Estado es una iniciativa engañosa que indica que el gobierno está volviendo a tomar control de la educación superior. En efecto, las nociones de autonomía y de rendición de cuentas son igualmente engañosas en la medida en que sugieren que la supervisión del rendimiento es el precio pagado por una mayor autonomía (Enders et al., 2013). Por consiguiente, se puede establecer que en el contexto chileno, el financiamiento público ha buscado una mayor racionalidad económica vinculando los fondos con los resultados, lo cual en Chile se da principalmente mediante tres instrumentos: Aporte Fiscal Directo (AFD), Aporte Fiscal Indirecto (AFI) y el Programa de Mejoramiento de la Calidad y el Desempeño de la Educación Superior (MECESUP), donde la experiencia de este último ha sido consonante en promover los objetivo de la política de aumentar el acceso y la equidad, incrementar la eficiencia externa e interna del sistema, al implementar en el año 2007 el instrumento Convenio de Desempeño, cuyo propósito general es incrementar la efectividad del gasto público, coordinar los objetivos institucionales con los de interés nacional, promover la rendición de cuentas públicas así como asociar la mejora del desempeño institucional con el financiamiento (Reich et al., 2011). Este piloto de convenio de desempeño se basaba en un contrato firmado entre el Ministerio de Educación y las instituciones universitarias, sobre la base de un Plan de Mejoramiento Institucional acordado previamente, donde se definen los objetivos a lograr, las estrategias para alcanzar dichos objetivos medidos en indicadores de resultados (MINEDUC, 2015b).

Vale precisar que la idea de autonomía en la universidad fue reflejada a través de la tradición idealista de la universidad moderna, influenciada por la concepción que tenía la teoría social de la modernidad, la cual se basaba en la creencia de que esta institución requiere autonomía de una sustancial política o influencia corporativa para funcionar óptimamente, donde el Estado tenía el rol de guardián de éstas en cuestiones de fondo, garantizando el financiamiento estatal, al menos en Europa continental, el fortalecimiento profesional gobernanza y la libertad académica. Como es de esperar los recursos financieros (riqueza de la nación, financiamiento de la educación superior y presupuestos de las universidades) llegan a ser un fuerte predictor de la cantidad de producción de los procesos principales de las universidades (Enders et al., 2013).

Por consiguiente, este tipo de financiamiento de convenio de desempeño se circunscribe dentro de los instrumentos de financiamiento público existentes que hacen referencia a traspasos de recursos o de fondos concursables mediante proyectos específicos (Yutronic et al., 2012). En este sentido, los convenios de desempeño, se configuran como una herramienta de adjudicación de recursos que se materializa de 
manera competitiva, donde se busca lograr impactos estratégicos, y que por tanto se entrega en base a resultados concretos, con lo cual se permite a las instituciones generar iniciativas de cambio, y que con ello puedan hacer frente a los problemas estructurales de diversa naturaleza y focalizar los esfuerzos institucionales en áreas vinculados al mejoramiento de la calidad de su quehacer (MINEDUC, 2015a). De igual modo, esta nueva forma de gestión devela a su vez uno de los desafíos más relevantes en el gobierno de las organizaciones de educación terciaria, el cual hace referencia a cómo poder establecer un contrato que sea lo más eficiente posible entre dos partes, principal y agente, de tal modo que permita conseguir un equilibrio en esta relación del gobierno y la sustentabilidad de la institución (Ganga y Valdivieso, 2013).

Mientras las presiones políticas por instituciones de educación superior eficientes y responsables han sido importantes, los territorios de liderazgo y gobernanza se vinculan en una interfaz entre el mundo interior y exterior (Middlehurst, 1999). Es así como en Francia, el centro del financiamiento consiste en tres partes: financiamiento de personal, de actividades de investigación y de actividades no investigativas (Kaiser y Neave, 2015). Como consecuencia a lo señalado anteriormente, y con el fin de reducir los obstáculos sobre el financiamiento por desempeño en las universidades, los Estados debieran considerar el mejoramiento de la capacidad que tienen estas instituciones de participar en el aprendizaje organizativo, entregando fondos y asistencia para la innovación organizacional requerida en el contexto del rol de las instituciones de educación superior (Pedraja-Rejas y Rodríguez-Ponce, 2014). Por otro lado resulta central abordar la resistencia institucional a la forma de financiamiento vía convenios de desempeño, creando mejores indicadores y medidas (Dougherty et al., 2013).

De igual forma, se debe tener en consideración que esta lógica de desempeño y de rendición de cuentas, no debe ser visualizada como algo únicamente externo, sino que también se deben percibir los beneficios más perdurables al interior de la institución, en la medida en que la responsabilidad, la transparencia y la integridad sean parte de la cultura organizacional, donde desde esta perspectiva no exista contradicción entre la rendición de cuentas y la autonomía, pues esta última hace referencia a la libertad académica y la primera se remite a la capacidad que tiene la universidad de administrar sus recursos hacia los objetivos que defina (Rojas y Bernasconi, 2009).

\section{CONCLUSIONES}

A partir del análisis conceptual llevado a cabo sobre el estado del arte en lo que respecta al financiamiento por desempeño y la rendición de cuentas, se plantea que este es un proceso y una tendencia de política pública que se encuentra a nivel mundial, que viene dado debido a fenómenos tales como la globalización, la internacionalización y el transnacionalismo imperantes en la actualidad. En este sentido, no se puede dejar fuera a esta corriente el contexto chileno de educación superior, ya que las instituciones nacionales no se encuentran ajenas a los cambios experimentados por las universidades a nivel mundial, configurándose entonces el financiamiento por desempeño como un instrumento que llegó para quedarse en nuestro país.

Por otra parte, no obstante el incremento del gasto público en educación superior, este sigue siendo bastante reducido en comparación a los países de la OCDE, lo cual lleva al replanteamiento y al análisis del rol que tiene hoy en día el financiamiento por parte del Estado, junto con la redefinición de la relación con las universidades, pretende incentivar que estas sean más rentables y eficientes en la utilización y destino de los recursos asignados, generando instrumentos de financiamiento que se rijan bajo la lógica del desempeño y de la rendición de cuentas, los cuales a su vez son asignados de manera competitiva entre las instituciones, conllevando esto a una necesaria reflexión en torno a cómo las universidades chilenas enfrentan este desafío, y el impacto que puede tener en la gestión y gobernanza institucional y en este sentido, respecto a la libertad de acción que se tiene sobre los recursos asignados. Ciertamente, este trabajo presenta una discusión teórica respecto a los conceptos generales asociados a esta nueva realidad.

Por otra parte, y considerando la experiencia internacional en el análisis de los convenios de desempeño, se destaca la importancia de poner en valor y rescatar los procesos asociados para el cumplimiento de las metas y objetivos y no centrarse únicamente en los resultados y análisis de los mismos.

Por último, se debe destacar que sin dudas el año 2015 marcará un punto de inflexión en las decisiones de financiamiento en el ámbito de la educación superior, dada la discusión existente respecto a la reforma que se pretende llevar a cabo para concretar la gratuidad, situación que deberá ser también considerada en los análisis posteriores de financiamiento por desempeño.

\section{AGRADECIMIENTOS}

Este trabajo cuenta con el patrocinio del CONICYT a través del financiamiento del Proyecto FONDECYT 1140026 y cuenta con el apoyo del Centro de Estudios de la Universidad de Tarapacá CEUTA. 


\section{REFERENCIAS}

Alexander, F. K., The changing face of accountability: Monitoring and assessing institutional performance in higher education. Journal of Higher Education, 411-431 (2000)

Altbach, P. G., Globalization and forces for change in higher education. In The international imperative in higher education (pp. 7-10). SensePublishers (2013)

Araneda-Guirriman, C.; E. Rodríguez-Ponce, y L. Pedraja-Rejas, Relación entre el Financiamiento Fiscal, la Calidad del Cuerpo Académico y la Retención de Estudiantes Universitarios en Chile, Formación universitaria: 6(6), 55-64 (2013)

Arata, A., y E. Rodríguez-Ponce, Desafíos de la dirección estratégica de las instituciones universitarias. Introducción de los editores. In Arata A, Rodríguez-Ponce E (Eds.) Desafíos y Perspectivas de la Dirección Estratégica de las Instituciones Universitarias. Pp, 23-32 (2009)

Barr, N., Higher education funding. Oxford review of economic policy: 20(2), 264-283 (2004)

Brunner, J. J., Políticas y mercados de educación superior: necesidades de información, in Brunner, JJ y $P$. Meller (comps.) Oferta y Demanda de Profesionales y Técnicos en Chile. Santiago de Chile: Ril Editores, 1985 (2004)

Brunner, J. J.; G. Elacqua; A. Tillett; J. Bonnefoy; S. González; P. Pacheco; F. Salazar. Guiar el mercado. Informe sobre la educación superior en Chile (2005).

Brunner, J. J., Medio siglo de transformaciones de la educación superior chilena: Un estado del arte, in A. Bernasconi (Ed.). La educación superior en Chile. Transformación, desarrollo y crisis, Colección de Estudios en Educación superior. CEPPE. Ediciones UC. 21-108 (2015)

de Boer, H.; B. Jongbloed; P. Benneworth; L. Cremonini, R. Kolster; A. Kottmann; H. Vossensteyn, Performance-based funding and performance agreements in fourteen higher education systems. Center for Higher Education Policy Studies (2015)

Dougherty, K. J.; S. Jones; H. Lahr; R. Natow; L. Pheatt y V. Reddy, Performance Funding for Higher Education Forms, Origins, Impacts, and Futures. The ANNALS of the American Academy of Political and Social Science, 655(1), 163-184 (2014)

Dougherty, K.; R. Natow, R. Bork; S. Jones y B. Vega, Accounting for higher education accountability: Political origins of state performance funding for higher education. Teachers College Record: 115(1), 1-50 (2013)

Dougherty, K. J., y V. Reddy, Performance Funding for Higher Education: What Are the Mechanisms What Are the Impacts: ASHE Higher Education Report, 39: 2. John Wiley y Sons (2013)

Enders, J.; H. De Boer y E. Weyer, Regulatory autonomy and performance: The reform of higher education re-visited, Higher education: 65(1), 5-23 (2013)

Fernández, E., Políticas públicas de educación superior desde 1990 hasta el presente. En Bernasconi, A. (Ed.) La educación superior de Chile. Trasnformación, desarrollo y crisis. 173-217. Ediciones UC (2015)

Flores, L.; J. González; J. Rodríguez; D. Sugg, D. Aporte fiscal indirecto: una propuesta para su modernización. Calidad en la educación, (35), 193-228 (2011)

Ganga, F., y P. Valdivieso, Innovación en el Gobierno y la Gestión Universitaria desde Nuevas Teorías y Prácticas Organizacionales: Una Reflexión desde la Teoría de Agencia y New Managerialism. Current Opinion in Creativity, Innovation and Entrepreneurship, 2(1): 21-27 (2013)

Laband, D.; B. Lentz, Do costs differ between for-profit and not-for-profit producers of higher education?. Research in Higher Education, 45(4), 429-441 (2004)

Laband, D.; B. Lentz, New estimates of economies of scale and scope in higher education. Southern Economic Journal 70(1): 172-183 (2003)

Lebeau, Y.; R. Stumpf; R. Brown; M. Lucchesi y M. Kwiek, M., Who shall pay for the public good? Comparative trends in the funding crisis of public higher education. Compare: A Journal of Comparative and International Education, 42(1), 137-157 (2012) 
Lopez, D.; J. Prieto; R. Reich; E. Rodríguez; J. Yutronic, Regulations and Challenges of Implementing Performance-based Agreements In Tertiary Education in Chile. Departamento de Financiamiento institucinal, División de Educación Superior, Ministerio de Educación de Chile, 18 p. (2011)

Hillman, N; D. Tandberg; y A. Fryar. Evaluating the Impacts of "New" Performance Funding in Higher Education. Educational Evaluation and Policy Analysis, (2015)

Hölttä, S.; P. Malkki. Response of Finnish higher education institutions to the national information society programme. Higher Education Policy, 13(3), 231-243 (2000)

Kaiser F. y G. Neave, Higher education policy in France. In Kaiser, F., Maassen, P., Meek, L., van Vught, F., de Weert, E., y Goedegebuure, L. (Eds.). (2014). Higher education policy: An international comparative perspective. Elsevier (2015)

Klein, S., Using Performance-Based Funding to Incentivize Change (RTI Press publication OP-0020-1501). Research Triangle Park, NC: RTI Press (2015)

Kretek, P.; Z., Dragšić y B. Kehm, Transformation of university governance: on the role of university board members. Higher Education, 65(1): 39-58 (2013)

Miao, K. Performance-Based Funding of Higher Education: A Detailed Look at Best Practices in 6 States. Center for American Progress (2012)

Middlehurst, R., Changing internal governance: are leadership roles and management structures in United Kingdom universities fit for the future?. Higher Education Quarterly, 67(3): 275-294 (2013)

Middlehurst, R., New realities for leadership and governance in higher education?. Tertiary Education \& Management, 5(4): 307-329 (1999)

MINEDUC, Financiamiento Institucional (en la web: http://www.mecesup.cl/) (2015a). Acceso 1 de diciembre 2015

MINEDUC, Análisis de indicadores educativos de Chile y la OCDE en el contexto de la Reforma Educacional. Serie evidencias 31 (2015b)

Nisar, M. A. Higher education governance and performance based funding as an ecology of games. Higher Education, 69(2), 289-302 (2015)

Paredes, R. Desafíos de la experiencia de financiamiento de la educación superior en Chile. En Bernasconi, A. (Ed.) La educación superior de Chile. Transformación, desarrollo y crisis. 219-257. Ediciones UC (2015)

Pedraja-Rejas, L., y E. Rodríguez-Ponce, El aseguramiento de la calidad: un imperativo estratégico en la educación universitaria. Ingeniare. Revista chilena de ingeniería, 23(1), 4-5 (2015)

Pedraja-Rejas, L., y E. Rodríguez-Ponce, Desafíos y roles de la educación superior. Ingeniare. Revista chilena de ingeniería, 22(2): 156-157 (2014)

Pettersen, I. J., From Metrics to Knowledge? Quality Assessment in Higher Education, Financial Accountability y Management, 31(1): 23-40 (2015)

Rabovsky, T. M., Accountability in higher education: Exploring impacts on state budgets and institutional spending patterns. Journal of Public Administration Research and Theory, 22(4): 675-700 (2012)

Reich, R., MECESUP Program in Chile 15 Years Supporting Higher Education Quality Improvement. Revista Harvard Review of Latin America Fall, 32-34 (2012)

Reich Albertz, R.; F. Machuca; D. López Stefoni; J. Prieto, J. P., Music, J., Rodríguez-Ponce, E., y Yutronic, J.. Bases y desafíos de la aplicación de convenios de desempeño en la educación superior de Chile. Ingeniare. Revista chilena de ingeniería, 19(1), 08-18 (2011)

Reiff, M., How to pay for public education. Theory and Research in Education, 12(1): 4-52 (2014)

Rodríguez Ponce, E.; C. Araneda Guirriman; L. Pedraja Rejas y J. Rodríguez Ponce, La Relación entre la Calidad de las Universidades y su Eficacia Económica: Un Estudio Empírico en Instituciones de Chile. Panorama Socioeconómico, (43): 79-89 (2011)

Rojas, A., y A. Bernasconi, El gobierno de las universidades en tiempos de cambio. In Arata A, RodríguezPonce E (Eds.) Desafíos y Perspectivas de la Dirección Estratégica de las Instituciones Universitarias. Pp. 183-214 (2009) 
Stensaker, B., y L. Harvey (Eds.), Accountability in Higher Education: Global Perspectives on Trust and Power. Routledge (2011)

Strehl, F.; S. Reisinger; M. Kalatschan, Funding systems and their effects on higher education system. OECD Education Working Papers, N 6, OECD Publishing (2007)

Viaene, J. y I. Zilcha, Public funding of higher education. No. 3606. CESifo working paper: Economics of Education, (2011)

Williamson, C.; J. Sánchez, Financiamiento universitario: principios básicos para el diseño de una politíca pública en Chile. Temas de la agenda pública 4(34), 30p (2009)

Wörner, C. Cumplimiento de metas: El caso de un incentive a las universidades chilenas parcialmente financiadas por el Estado. Archivos analíticos de políticas educativas, 22(73) (2014)

Yutronic, J.; R. Reich Albertz; E. Rodriguez Ponce; J. Music, y D. López Stefoni, Convenios de desempeño y su porte en el financiamiento de la Educación Superior en Chile. Educación Superior y Sociedad, 16(1): 53-77 (2012) 